\title{
Clustering for Security Challenges
}

\author{
Rakesh M. Verma \\ University of Houston \\ Houston, Texas \\ rverma@uh.edu
}

\author{
Srivathsan Srinivasagopalan \\ Alienvault Inc \\ Austin, TX \\ ssrinivasagopalan@alienvault.com
}

\begin{abstract}
Data science, which includes statistics, probability, data mining, machine learning and natural language processing, is on a major uptrend. Clustering is a key data science method, which does not require labeled data. This tutorial will cover clustering for security challenges. We expect to cover both classical clustering techniques and some of the important, newer clustering algorithms. Examples will be drawn from clustering of malware datasets and phishing emails. Throughout the discussion, we will highlight some of the key unique needs of the security domain that are relevant for clustering techniques.
\end{abstract}

\section{KEYWORDS}

unsupervised learning; clustering; unique needs of security domain; nonstationarity; tutorial

\section{ACM Reference Format:}

Rakesh M. Verma and Srivathsan Srinivasagopalan. 2019. Clustering for Security Challenges. In Fifth ACM International Workshop on Security and Privacy Analytics (IWSPA '19), March 27, 2019, Richardson, TX, USA. ACM, New York, NY, USA, 2 pages. https://doi.org/10.1145/3309182.3309184

\section{INTRODUCTION}

Data science is exploding as a field, e.g., University of Houston (UH) recently created a new Data Science Institute, which received \$10 million from a company to start a data science degree. Researchers have been applying data mining and machine learning techniques to security challenges for the past 15 years or so. A search of the bibliographic database $\mathrm{DBLP}^{1}$ with the query intrusion detection neural network led to 190 matches on January 28, 2019. Note that this result is for just one machine learning technique, viz., neural network, and just one security challenge, viz., intrusion detection. The mind boggles when one considers the plethora of data mining and machine learning techniques and the variety of security challenges, which have no good separations between legitimate instances and attack instances. These include phishing, malware, stepping-stone detection, intrusion detection, and denial of service attacks, just to name a few.

However, other researchers have observed that the overwhelming majority of this research is simply not being deployed [5]. So the question arises [6]: "Why is there such a gap between theory

\footnotetext{
${ }^{1}$ https://dblp.uni-trier.de/

Permission to make digital or hard copies of part or all of this work for personal or classroom use is granted without fee provided that copies are not made or distributed for profit or commercial advantage and that copies bear this notice and the full citation on the first page. Copyrights for third-party components of this work must be honored.

For all other uses, contact the owner/author(s).

IWSPA '19, March 27, 2019, Richardson, TX, USA

(C) 2019 Copyright held by the owner/author(s).

ACM ISBN 978-1-4503-6178-1/19/03.

https://doi.org/10.1145/3309182.3309184
}

and practice in security?" The reason for this gap seems to be that researchers, in their work, have missed at least some of the unique needs of the security domain. For a discussion on these aspects, see $[1,6,7]$.

Clustering is an important data analysis technique, with several books being solely devoted to this topic and its myriad applications. It has some key advantages, e.g., it does not require labeled data, as well as some pitfalls. We feel that cyber security researchers will benefit from exposure to some of the latest developments in the field, which have still not percolated into the classical text books on data mining and machine learning. Hence, we propose a tutorial on this topic for cyber security researchers and professionals. Next, we give an outline of the tutorial keeping in mind the most pertinent needs of the security domain.

\section{OUTLINE OF THE TUTORIAL}

This two-hour tutorial on clustering for security challenges will expose the audience to the following topics:

(1) What is clustering, why is it useful?

(2) Unique needs of security domain relevant to clustering: nonstationarity arising from an active attacker or streaming data; lack of large, diverse and representative datasets; potential poisoning of datasets, etc.

(3) Basic terminology and historical development of the clustering field.

(4) Different kinds of clusterings

(a) Partitional clustering/Hierarchical clustering/Probabilistic Model-based Methods/Spectral

(b) Exclusive/Overlapping/Fuzzy

(c) Complete/Partial

(5) Different kinds of clusters: prototype-based, graph-based, etc.

(6) Classical clustering algorithms (e.g., K-means and its variants, hierarchical, DBScan and its variants)

(7) Recent developments in clustering

(8) Evaluation of clustering

(9) How to pick the number of clusters for methods that require it as an input?

(10) Parallel and distributed clustering

(11) Limitations of clustering

(12) Best practices for clustering

If time permits, we will also look at incremental clustering techniques and the case of streaming data. Recently, so-called supervised clustering methods have been developed. As mentioned above, clustering is a vast topic with many books and chapters in data mining books. We will draw inspiration for this tutorial from a variety of sources. Most of the clustering methods will be illustrated with material drawn from our recent work on malware clustering [2, 3] 
and related sources, and also our from recent work on clustering for phishing email datasets by other researchers [8] and us [4].

\section{INTEGRATION IN A SECURITY ANALYTICS COURSE}

A Security Analytics course has been offered at UH at the senior undergraduate and introductory graduate levels since Spring 2015 It was approved as a regular course at the graduate level in 2016 and at the undergraduate level in 2017.

The course follows a modular format in which there are four modules: basics of security, unsupervised learning techniques for security, supervised learning techniques for security, and natural language processing for security. In the unsupervised learning module, we deal with association rule mining and clustering. Material from this tutorial will be used for the discussion on clustering in this course.

\section{SHORT BIOGRAPHY OF TUTORIAL PRESENTERS}

Rakesh Verma is a Professor of Computer Science at the University of Houston. He received his Ph.D. in Computer Science with a dissertation on efficient algorithms for equational logic programming. Since then he has conducted research on natural language processing, cryptographic protocol verification, unsupervised and supervised techniques for phishing URL, website and email detection, email masquerade attacks and malware clustering. He has been teaching a course on security analytics at UH since 2015.

Srivathsan Srinivasagopalan is a Machine Learning Researcher at AlienVault Inc / AT\&T Cybersecurity based in Austin, TX. He has a Ph.D. in theoretical Computer Science and has been developing algorithms for breach detection, correlation and infusing intelligence into various aspects of the organization using machine learning and AI techniques for about eight years. Previously, he has held lead roles at various startups and large enterprises doing machine learning + cybersecurity.

\section{ACKNOWLEDGMENTS}

Thanks to NSF for partial support under grants DUE 1241772, CNS 1319212, DGE 1433817, and DUE 1356705. This material is also based upon work supported by, or in part by, the U. S. Army Research Laboratory and the U. S. Army Research Office under contract/grant number W911NF-16-1-0422.

\section{REFERENCES}

[1] Idan Amit, John Matherly, William Hewlett, Zhi Xu, Yinnon Meshi, and Yigal Weinberger. 2018. Machine Learning in Cyber-Security - Problems, Challenges and Data Sets. arXiv e-prints, Article arXiv:1812.07858 (Dec. 2018), arXiv:1812.07858 pages. arXiv:cs.LG/1812.07858

[2] Houtan Faridi, Srivathsan Srinivasagopalan, and Rakesh Verma. 2018. Performance Evaluation of Features and Clustering Algorithms for Malware. IEEE ICDMW (ADMiS) (2018).

[3] Houtan Faridi, Srivathsan Srinivasagopalan, and Rakesh Verma. 2019. Parameter Tuning and Confidence Limits of Malware Clustering. In Proceedings of the Ninth ACM on Conference on Data and Application Security and Privacy (CODASPY '19).

[4] Vibhu Sharma. 2018. Trend Analysis On Phishing Email Data Using Natural Language Processing. Master's thesis. University of Houston, Houston, TX, 77204, USA.

[5] Robin Sommer and Vern Paxson. 2010. Outside the Closed World: On Using Machine Learning for Network Intrusion Detection. In 31st IEEE Symposium on Security and Privacy, S\&P 2010, 16-19 May 2010, Berleley/Oakland, California, USA. 305-316.

[6] Rakesh Verma. 2018. Security Analytics: Adapting Data Science for Security Challenges. In Proceedings of the Fourth ACM International Workshop on Security and Privacy Analytics (IWSPA '18). ACM, New York, NY, USA, 40-41. https: //doi.org/10.1145/3180445.3180456

[7] Rakesh M. Verma, Murat Kantarcioglu, David J. Marchette, Ernst L. Leiss, and Thamar Solorio. 2015. Security Analytics: Essential Data Analytics Knowledge for Cybersecurity Professionals and Students. IEEE Security \& Privacy 13, 6 (2015), 60-65.

[8] John Yearwood, Dean Webb, Liping Ma, Peter Vamplew, Bahadorreza Ofoghi, and Andrei Kelarev. 2009. Applying Clustering and Ensemble Clustering Approaches to Phishing Profiling. In Proceedings of the Eighth Australasian Data Mining Conference - Volume 101 (AusDM '09). Australian Computer Society, Inc., Darlinghurst, Australia, Australia, 25-34. http://dl.acm.org/citation.cfm?id=2449360.2449369 